\title{
Electrochemical Microbiosensor for Detecting COVID-19 in a Patient Sample Based on Gold Microcuboids Pattern
}

\author{
Waleed A. El-Said ${ }^{1}$ (1) - Abdullah S. Al-Bogami ${ }^{1}$ - Wael Alshitari ${ }^{1}$ - Deia A. El-Hady ${ }^{1} \cdot$ Tamer S. Saleh $^{1}$. \\ Mohamed A. El-Mokhtar ${ }^{2} \cdot$ Jeong-Woo Choi ${ }^{3}$
}

Received: 6 April 2021 / Revised: 14 June 2021 / Accepted: 18 June 2021 / Published online: 9 August 2021

(c) The Korean BioChip Society 2021

\begin{abstract}
As continues increasing the COVID-19 infections, there is an urgent need for developing fast, simple, selective, and accurate COVID-19 biosensors. A highly uniform gold ( $\mathrm{Au}$ ) microcuboid pattern was used as a microelectrode that allowed monitoring a small analyte. The electrochemical biosensor was used to monitor the COVID-19 S protein within a concentration range from 100 to $5 \mathrm{pmol} \mathrm{L}^{-1}$; it showed a lower detection limit of $276 \mathrm{fmol} \mathrm{L}^{-1}$. Finally, the developed COVID-19 sensor was used to detect a positive sample from a human patient obtained through a nasal swab; the results were confirmed using the PCR technique. The results showed that the SWV technique showed high sensitivity towards detecting COVID-19 and good efficiency for detecting COVID-19 in a positive human sample.
\end{abstract}

Keywords COVID-19 $\cdot$ Gold microelectrode $\cdot$ Cyclic voltammetry $\cdot$ Square wave voltammetry $\cdot$ Electrochemical biosensor $\cdot$ Raman spectroscopy $\cdot$ Nasal swab

\section{Introduction}

More than 30 strains of coronavirus were identified, including HCoV-229E, HCoV-OC43, SARS-CoV, HCoV-NL63, HCoV-HKU1, and MERS-CoV [1]. In addition, a novel coronavirus (COVID-19) has reported at the end of 2019, which has fast spread worldwide in a short time compared with other coronaviruses [2-6]. Thus, there is an urgent need to find antiviral drugs besides developing an accurate, simple, and sensitive sensing method. However, the early diagnosis is difficult because there are no initial characteristic symptoms of COVID-19 in the early stage of infection; besides, many cases did not show the usual signs [7].

Waleed A. El-Said

waahmed@uj.edu.sa

Jeong-Woo Choi

jwchoi@ sogang.ac.kr

1 Department of Chemistry, College of Science, University of Jeddah, P.O. Box 80327, Jeddah 21589, Saudi Arabia

2 Department of Microbiology and Immunology, Faculty of Medicine, Assiut University, Assiut 71515, Egypt

3 Department of Chemical and Biomolecular Engineering, Sogang University, 35 Baekbeom-Ro, Mapo-Gu, Seoul 04107, Republic of Korea
The PCR assay is the golden technique for COVID-19 detection; however, the high cost, needing specialist laboratories, skilled persons, and consuming a long time are the main challenges for its widely uses [8,9]. On the other hand, the enzyme-linked immunosorbent assay (ELISA) was used to detect the receptor protein using the corresponding antibody $[10,11]$. Thus, it is of great urgency to develop rapid, reproducible, cost-effective, easy-to-use, accurate, sensitive, and selective assays for the early diagnosis of COVID-19 in different specimens.

Biosensors possess many advantages, including the fast response and the high selectivity towards several biological species. Electrochemical-based biosensors are among the most widely used, simple, accurate, prompt, and sensitive detecting assays of biomarkers without any pretreatments with a very low detection limit (LOD) [11-13]. Nanomaterials (e.g., gold and silver) were reported to modify the traditional electrodes to develop highly sensitive and selective electrochemical sensors [14-22].

We reported developing a sensitive and selective COVID19 electrochemical biosensor using a highly uniform $\mathrm{Au}$ micropattern to improve the electrochemical conductivity. The anti-COVID-19 antibodies were used as probes for monitoring the COVID-19 based on cyclic voltammetry (CV) and square wave voltammetry (SWV) techniques. The 
developed biosensor showed the capability to detect the COVID-19 protein over a wide range of concentrations from $5 \mathrm{nmol} \mathrm{L}{ }^{-1}$ to $100 \mathrm{pmol} \mathrm{L}^{-1}$ with a LOD of $276 \mathrm{fmol} \mathrm{L}^{-1}$ SWV technique. Furthermore, we have used this biosensor to detect the COVID-19 in a real human sample obtained from the nasal swab, which confirmed the capability of this electrochemical biosensor for real uses for detecting the COVID-19 without any sample preparations.

\section{Results and Discussion}

\subsection{Characterization of the Au Micropattern Substrate}

Figure 1a showed a schematic diagram of the COVID-19 electrochemical biosensor based on CV and SWV techniques. The sensor is composed of Au microcuboid patterncoated PDMS as microelectrode. Firstly, we immobilized the
anti-COVID-19 antibody onto the Au microcuboid pattern was used it as a specific probe for COVID-19 S protein to capture the COVID-19 protein. Furthermore, the Au microcuboid pattern would improve the electrochemical conductivity and analyze a small target analyte. Next, the SEM image was used to investigate the morphology of the $\mathrm{Au}$ micropattern substrate that indicated the highly uniform distribution of the Au cuboid structures with a length of about $1.47 \mu \mathrm{m}$ and a width of about $730 \mathrm{~nm}$ as shown in Fig. 1b. Furthermore, the topography of this substrate was studied using the AFM technique, which showed the fabrication of a uniform microcuboid pattern with a length of about $1 \mu \mathrm{m}$ and a width of about $500 \mathrm{~nm}$ (Fig. 1c).

\subsection{Electrochemical Detection of COVID-19 Protein}

The direct immobilization of $50 \mu \mathrm{M}$ of anti-COVID-19 antibody onto the Au cuboid microcuboid was confirmed using Raman spectroscopy. The SERS spectrum of the (a)

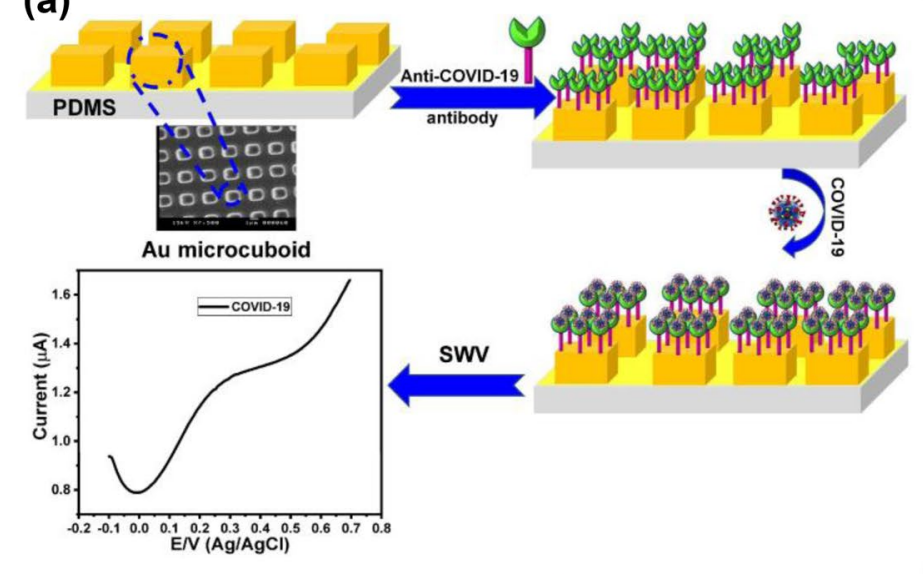

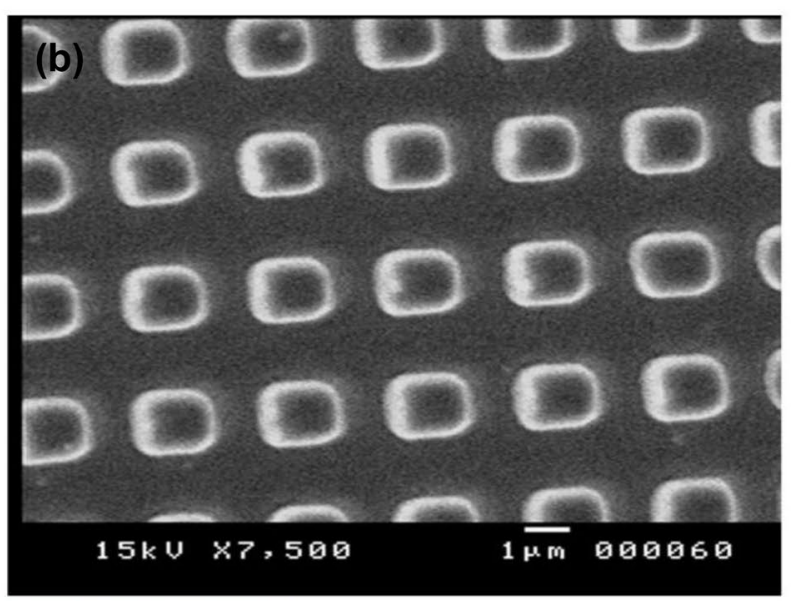

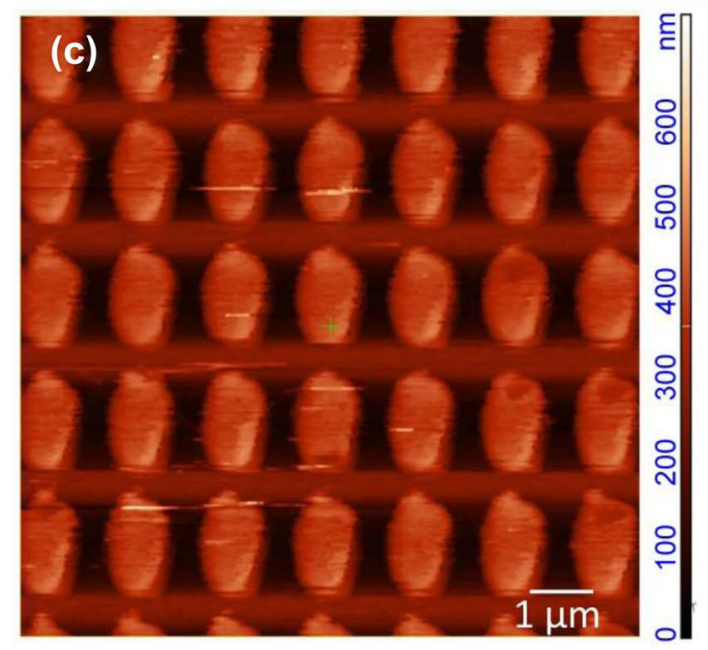

Fig. 1 a Schematic diagram of electrochemical COVID-19 microbiosensor that including, (i) immobilization of antiCOVID-19 antibody, and (ii) capturing of COVID-19 protein (not real scale), b SEM image of Au microcuboid structures, scale bar $1 \mu \mathrm{m}$ and (c) AFM topography of the Au microcuboid structures, scale bar $500 \mathrm{~nm}$ 
anti-COVID-19 antibody was represented in Fig. 2a, which showed a set of SERS peaks located at $541 \mathrm{~cm}^{-1}(\mathrm{~S}-\mathrm{S}$ bond of protein), $635 \mathrm{~cm}^{-1}$ (Tyr: C-C twisting), $936 \mathrm{~cm}^{-1}$ $(\alpha$-helix, str. $\mathrm{C}-\mathrm{C}$, str. $\mathrm{C}-\mathrm{N}), 1070 \mathrm{~cm}^{-1}$ (str. $\mathrm{C}-\mathrm{N}$ in protein, phenylalanine), $1189 \mathrm{~cm}^{-1}$ (tryptophan, phenylalanine, str. $\mathrm{C}-\mathrm{N}$ and bending $\mathrm{N}-\mathrm{H}$ of Amide III), $1318 \mathrm{~cm}^{-1}$ ( $\alpha$-helix, Amide III), and $1444 \mathrm{~cm}^{-1}$ (Amide II/N-H bending, C-N stretch, bending $\mathrm{CH}_{2}$ of proteins) [23-28].

The COVID-19 biosensor was fabricated by interacting a $50 \mu \mathrm{L}$ of the COVID-19 protein with an anti-COVID-19 antibody/Au microcuboid pattern. Figure $2 \mathrm{~b}$ showed the SERS spectra of the anti-COVID-19 antibody/Au microcuboid substrate before and after the interaction with the COVID-19 protein, which exhibited a set of new SERS signals. The characteristics SERS signals including peaks at $526 \mathrm{~cm}^{-1}(\mathrm{~S}-\mathrm{S}), 655 \mathrm{~cm}^{-1}$ (tryptophan, sidechain/indole ring modes), $770 \mathrm{~cm}^{-1}$ (tryptophan, sidechain/indole ring modes), $927 \mathrm{~cm}^{-1}$ ( $\alpha$-helix, Backbone skeletal stretch/C-C, C-N stretches), $1032 \mathrm{~cm}^{-1}$ (phenylalanine, sidechain/phenyl ring modes), $1144 \mathrm{~cm}^{-1}$ and $1185 \mathrm{~cm}^{-1}$ (tyrosine and phenylalanine, sidechain/indole ring mode), $1320 \mathrm{~cm}^{-1}(\alpha$-helix, Amide III/C-N stretch, N-H deformation), $1420 \mathrm{~cm}^{-1}$
(Symmetric $\mathrm{COO}^{-}$stretch), $1456 \mathrm{~cm}^{-1}$ (Amide II/N-H bending, $\mathrm{C}-\mathrm{N}$ stretch), $1544 \mathrm{~cm}^{-1}$ and $1586 \mathrm{~cm}^{-1}$ (tryptophan, Amide II/indole ring mode), and $1676 \mathrm{~cm}^{-1}$ ( $\beta$-sheet, Amide $\mathrm{I} / \mathrm{C}=\mathrm{O}$ stretch).

After confirmation of the capture of COVID-19 protein by anti-COVID-19 anti-body immobilized on Au microcuboid electrode (Fig. 2b), the CV response of the substrate was investigated before and after immobilization of the COVID-19 protein. Figure 2c showed the cyclic voltammogram of the bare Au microcuboid electrode that represented a background without any redox peaks. The $\mathrm{CV}$ response of the an-ti-COVID-19 antibody/Au microcuboid electrode was investigated in Fig. 2d, which indicated two redox peaks, a cathodic peak at about $0.0 \mathrm{~V}$ and a weak and broad oxidation peak. Thus, it is difficult to determine the oxidation potential peak. Figure 2e showed the cyclic voltammogram of the anti-COVID-19 anti-body/Au microcuboid electrode after capturing $50 \mu \mathrm{L}$ of COVID-19 protein. It illustrated two redox peaks, a cathodic peak at about $0.27 \mathrm{~V}$ and a. oxidation peak at about $0.45 \mathrm{~V}$.

The cyclic voltammograms of different concentrations of COVID-19 protein were recorded and represented in
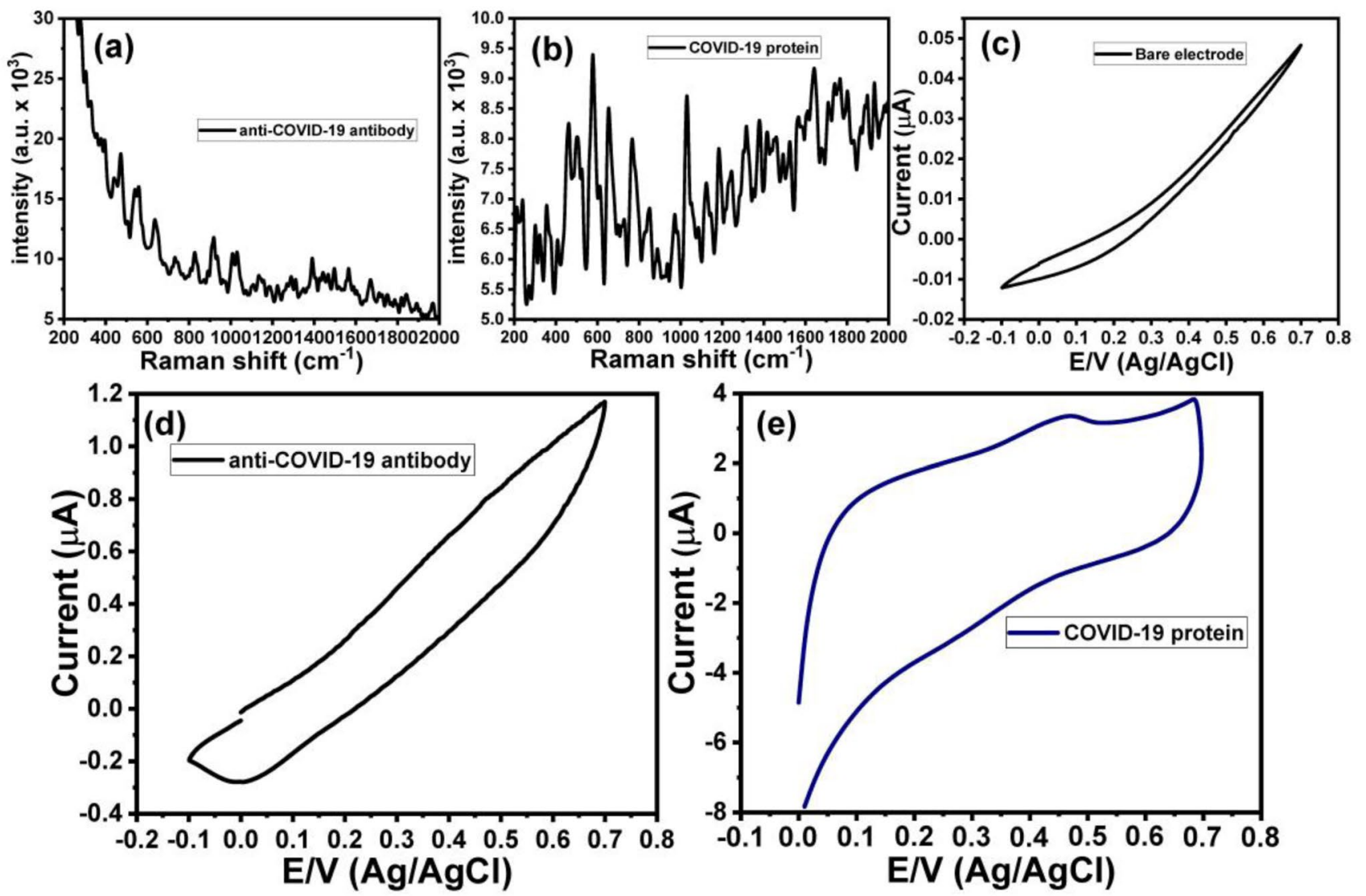

Fig. 2 a SERS spectrum of anti-COVID-19 antibody, b SERS spectra of COVID-19 protein in comparing after capturing with antiCOVID-19 antibody, $\mathbf{c}$ CV of bare Au microcuboid electrode, $\mathbf{d ~ C V}$

of anti-COVID-19 antibody/Au microcuboid electrode, and e CV of COVID-19 protein after interacting with anti-COVID-19 antibody/Au microcuboid electrode 
Fig. 3a to investigate the efficiency of the developed sensor. The results indicated the increase of the current peak with increasing the con-certation of the COVID-19 protein. Figure $3 b$ showed the relationship between the COVID-19 protein concentrations and the current peak at about $0.45 \mathrm{~V}$, which showed linear plots within a concentration range from $10 \mathrm{pmol} \mathrm{L}^{-1}$ to $100 \mathrm{nmol} \mathrm{L}^{-1}$ with a slope of $1.1, R^{2} 0.9813$, and LOD of $19.5 \mathrm{pmol} \mathrm{L}^{-1}$.

Furthermore, to develop a highly sensitive COVID-19based sensor, we have utilized the SWV technique that is more sensitive than the CV technique. Figure 4 a showed the SWV curve of the anti-COVID-19 antibody/Au microcuboid electrode within a potential window from -0.1 to $0.7 \mathrm{~V}$, which almost did not show any oxidation peak. On the other hand, the SWV voltammogram of the anti-COVID-19 antibody/Au microcuboid electrode after interaction with $100 \mathrm{pmol} \mathrm{L}^{-1}$ of COVID-19 protein showed an oxidation peak at about $0.45 \mathrm{~V}$ (Fig. 4b).

The effect of the scan rate on the electrochemical response of the developed sensor was studied in Fig. 4c, which shows the SWV voltammograms of $50 \mathrm{nmol} \mathrm{L}^{-1}$ of the COVID-19 protein under different scan rates (from 10 to $100 \mathrm{mV} / \mathrm{s}$ ). The results demonstrated that the redox current peaks were increased with increasing the potential scan rate; besides, the potential oxidation peak was shifted to the right side as increasing the potential scan rate. Furthermore, the relationship between the oxidation current peak and the square root of the scan rate showed a linear plot with an $R^{2}$ of 0.985 (Fig. 4d). The linear nature of the plot indicated that this system is reversible.

The efficiency of the developed sensor based on the SWV technique was also studied. Figure 5a showed the SWV voltammograms of anti-COVID-19 antibody/Au microcuboid electrode after interacting with different concentrations of COVID-19 protein within a range from 5 pmol L $\mathrm{L}^{-1}$ to $100 \mathrm{nmol} \mathrm{L}^{-1}$. The results showed an increase of the current peak as the concertation of the COVID-19 protein increased. Figure $5 \mathrm{~b}$ illustrated the relationship between the concentrations of COVID-19 protein and the corresponding current peak at about $0.45 \mathrm{~V}$ that showed linear plots within this range of concentrations with a slope of 3.22 and $R^{2}$ of 0.988 . The LOD of the developed sensor based on the SWV technique was found to be $276 \mathrm{fmol} \mathrm{L}^{-1}$.

To investigate the performance of the present sensor in comparison with the previously reported COVID-19 sensor. Table 1 summarized the values of the LOD for several COVID-19 sensors based on different detecting techniques. It is worth noting that the present sensor possesses a good LOD compared to most previously reported sensors. Although some reported sensors showed lower LOD than the presented sensor, this sensor's key advantages are its simplicity and fast detection time [29-37].

\subsection{Performance of the Developed Electrochemical COVID-19-Based Sensor for Monitoring COVID-19 in a Patient Sample}

Finally, we have used the developed sensor for monitoring the COVID-19 in a patient sample. At first, the presence of the COVID-19 in the sample was confirmed by using the PCR technique, which indicates that this sample having the RNA gene of COVID-19. Then, we have used the developed sensor for detecting the COVID-19 based on the CV and SWV techniques. Figure 6a showed the CV response of the COVID-19 sample with the antiCOVID-19 antibody/Au microcuboid electrode, which

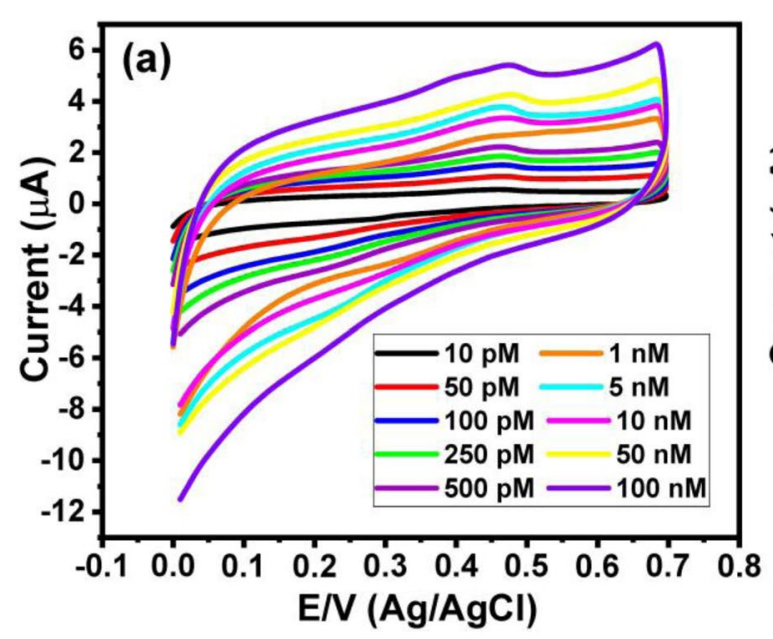

Fig. 3 a The CV of different concentrations of COVID-19 protein within a range from $100 \mathrm{nmol} \mathrm{L}{ }^{-1}$ to $5 \mathrm{pmol} \mathrm{L}^{-1}$ after interacting with anti-COVID-19 antibody/Au microcuboid electrode and $\mathbf{b}$ the

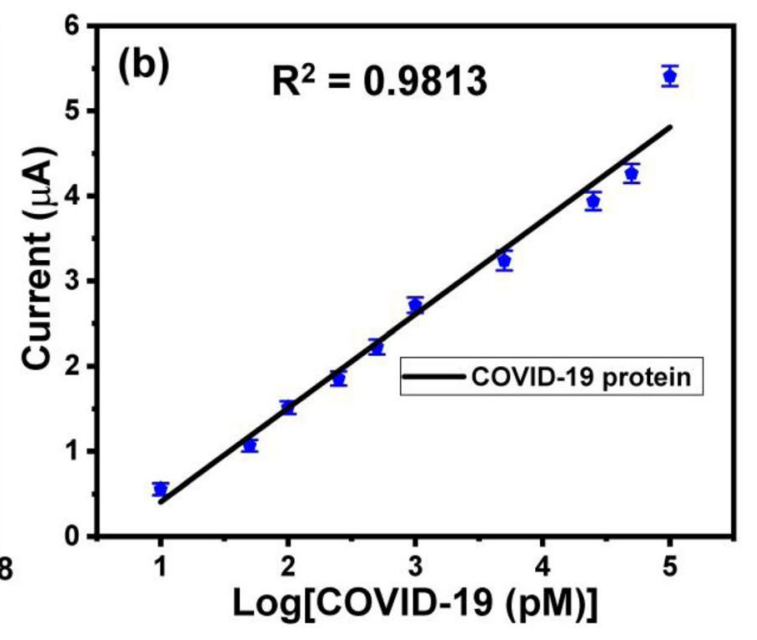

relationship between the oxidation current peak and the concentration of COVID-19 protein 

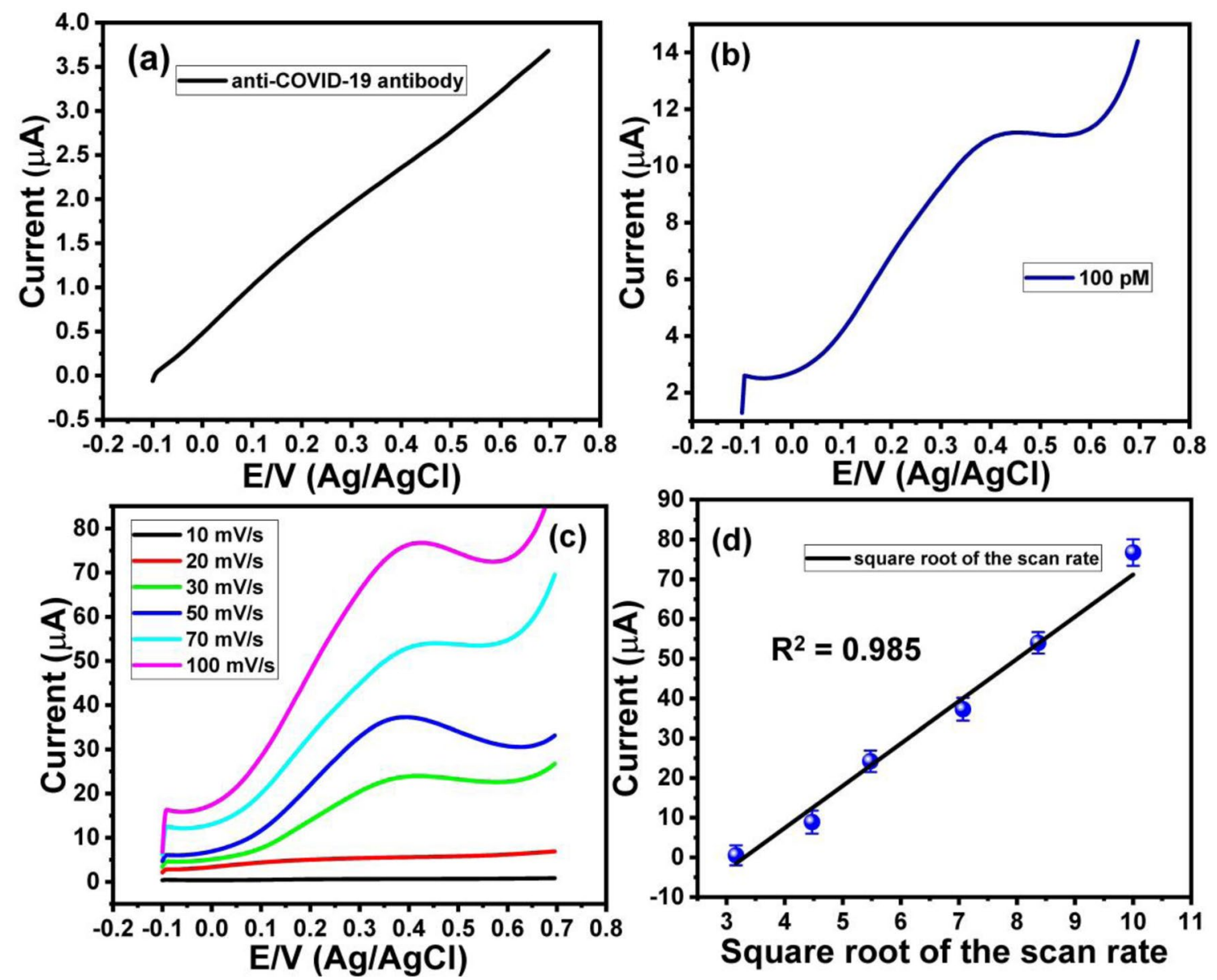

Fig. 4 a SWV voltammogram of anti-COVID-19 antibody/Au microcuboid electrode, b SWV voltammogram of COVID-19 protein after interacting with anti-COVID-19 antibody/Au microcuboid electrode, c the SWV voltammograms of COVID-19 protein after interacting

with anti-COVID-19 antibody/Au microcuboid electrode at different scan rate, and $\mathbf{d}$ relationship between the scan rate and the corresponding oxidation current peak

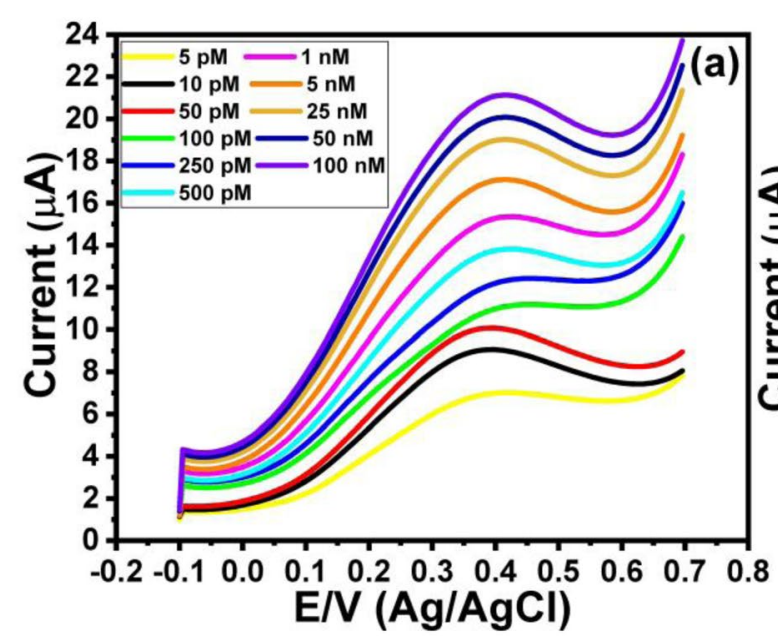

Fig. 5 a SWV voltammograms of different concentrations of COVID-19 protein within a range from $100 \mathrm{nmol} \mathrm{L}-1$ to $5 \mathrm{pmol} \mathrm{L}^{-1}$ after interacting with anti-COVID-19 antibody/Au microcuboid elec-

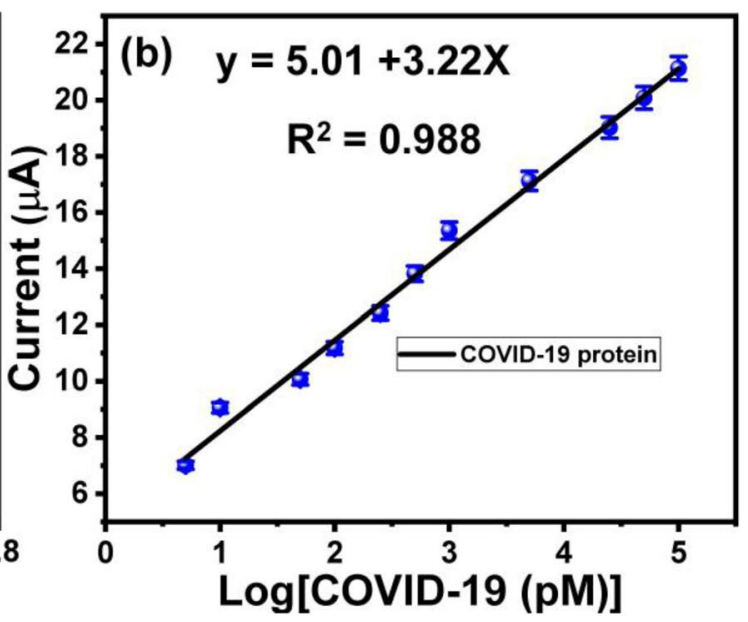

trode and $\mathbf{b}$ the relationship between the oxidation current peak and the concentration of COVID-19 protein 
Table 1 Comparison between the developed sensor and the previously reported COVID-19 sensors

\begin{tabular}{lll}
\hline Sensor & LOD & References \\
\hline Glyconanoparticle platform & $5 \mathrm{nmol} \mathrm{L}^{-1}$ & {$[29]$} \\
Oligonucleotide capped Plasmonic nanoparticles & $0.18 \mathrm{ng} \mathrm{H}^{-1}$ & {$[30]$} \\
DNA nanoscaffold hybrid chain reaction & $0.96 \mathrm{pmol} \mathrm{L}^{-1}$ & {$[31]$} \\
ELISA & $100 \mathrm{ng} \mathrm{mL}^{-1}$ & {$[32]$} \\
Quantum dot-based & $10 \mathrm{ng} \mathrm{mL}^{-1}$ & {$[32]$} \\
SARS-CoV-2 immunodiagnostics kit & $250 \mathrm{pg} \mathrm{mL}^{-1}$ & {$[33]$} \\
Electrochemical impedance spectroscopy (EIS) & $2.8 \mathrm{fmol} \mathrm{L}^{-1}$ & {$[34]$} \\
(Nanoprinted Reduced-Graphene-Oxide) & & \\
Optical-localized surface plasmon resonance (LSPR) & $2 \mathrm{nmol} \mathrm{L}^{-1}$ & {$[35]$} \\
Piezoelectric immunosensor & $3.5 \mathrm{ng} \mathrm{mL}^{-1}$ & {$[36]$} \\
Square wave voltammetry & $0.4 \mathrm{pg} \mathrm{mL}^{-1}$ & {$[37]$} \\
Square wave voltammetry & $276 \mathrm{fmol} \mathrm{L}^{-1}$ & This work
\end{tabular}
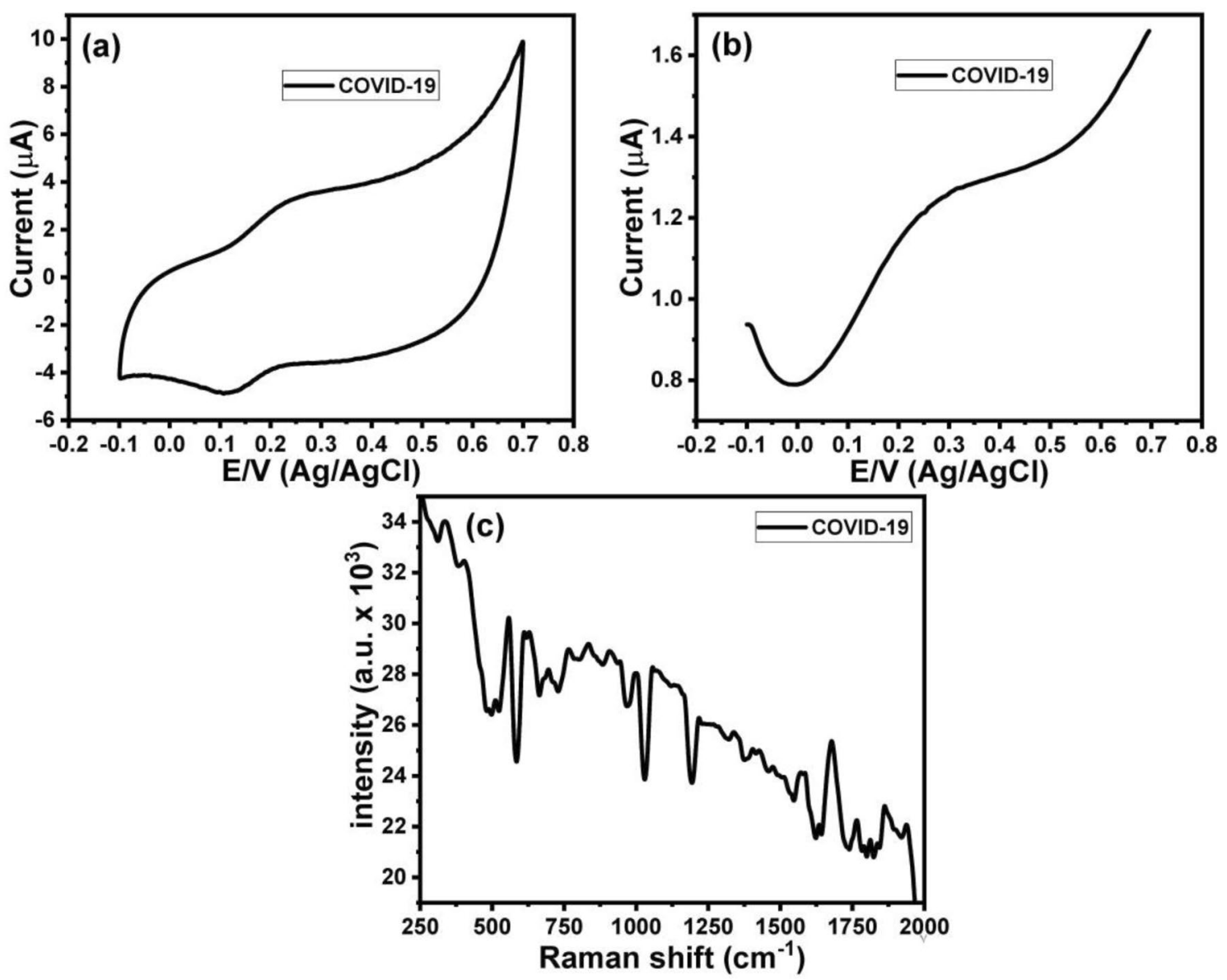

Fig. 6 a SERS spectra of COVID-19 protein admixed with human serum in compared with COVID-19 protein in PBS, b CV of COVID-19 virus after interacting with anti-COVID-19 antibody/Au microcuboid electrode, $\mathbf{c}$ SWV of COVID-19 virus after interacting

showed a revisable response with an oxidation peak at about $0.3 \mathrm{~V}$ and a cathodic peak at $0.1 \mathrm{~V}$. it is worth noting the difference between the response of the COVID-19 with anti-COVID-19 antibody/Au microcuboid electrode and d SERS spectrum of COVID-19 virus after interacting with anti-COVID-19 antibody/Au microcuboid electrode

than COVID-19 protein because of the difference between the whole virus rather than the $\mathrm{S}$ protein. Furthermore, we have studied the SWV response of the COVID-19 by 
using the developed sensor, which showed an oxidation peak at about $0.3 \mathrm{~V}$ (Fig. 6b).

To confirm the capture of the COVID-19 with the anti-COVID-19 antibody/Au microcuboid electrode, we have recorded the corresponding SERS spectrum (Fig. 6c), which exhibited a set of SERS signals, especially SERS peaks at Raman shifts of $372 \mathrm{~cm}^{-1}$, $518-533 \mathrm{~cm}^{-1}, 651 \mathrm{~cm}^{-1}, 724-838 \mathrm{~cm}^{-1}, 936 \mathrm{~cm}^{-1}$, $946 \mathrm{~cm}^{-1}, 1038 \mathrm{~cm}^{-1}, 1086 \mathrm{~cm}^{-1}, 1288 \mathrm{~cm}^{-1}, 1314 \mathrm{~cm}^{-1}$, $1413 \mathrm{~cm}^{-1}$, and $1596 \mathrm{~cm}^{-1}$. These data showed the shifting of some besides the disappearance of the SERS peak at $290 \mathrm{~cm}^{-1}$ upon the presence of COVID-19 protein in the human serum. The results demonstrated the efficiency of the developed sensor for detecting the COVID-19 protein in the presence of human serum and hence the capability to detect the COVID-19 in real samples. The selectivity of the SERS technique is related to the fact that the SERS spectrum showed a unique fingerprint spectrum for each biomolecule species. The high sensitivity of the developed sensor is attributed to the uses of the $\mathrm{Au}$ microcuboid structures, which enhance the SERS signals of the COVID-19.

\section{Conclusion}

A new Au microcuboid modified PDMS was used as a flexible microelectrode and acted as a scaffold for immobilization of anti-COVID-19 antibody. Uses of the microstructures allowed the detection of a small amount of the analyte and improved the developed sensor's electrochemical conductivity. Furthermore, the interaction between the anti-COVID-19 antibody and COVID-19 protein was confirmed based on the SERS technique. Moreover, the $\mathrm{CV}$ and SWV techniques were used to monitor different concentrations of the COVID-19 protein that showed high efficiency to monitor COVID-19 protein with a detection limit of $276 \mathrm{fmol} \mathrm{L}^{-1}$. Besides, we have successfully detected the COVID-19 virus in a real human sample. Thus, the developed sensor showed high sensitivity towards the COVID-19 protein and demonstrated the capability to direct COVID-19 in human samples without complicated sample preparation. One of the advantages of using this highly uniform Au nanoplate lies in using the highly uniform distribution of the micropattern with the same size and morphology that could enhance the data's reproducibility and result in quantitative data. In addition, this flexible substrate could open further applications such as chem/biosensors based on different techniques, including surface plasmon resonance, or as a SERS-active surface combined with metal nanoparticles.

\section{Materials and Methods}

\subsection{Materials and Reagents}

Au micropattern was obtained from Nanobioelectronics Laboratory (NBEL), Sogang University, Korea. PBS ( $\mathrm{pH}, 7.4$ ) was purchased from Sigma-Aldrich (St. Louis, MO, USA). COVID-19 antibody (S1N-M122, monoclonal antibody, IgG1) and COVID-19 S1 protein (S1N-C52H2, $\mathrm{MWt}=76.9 \mathrm{kDa}$ ) were purchased from ACRO Biosystems (Newark, Delaware, USA). All other chemicals were analytical-grade reagents.

\subsection{Fabrication of the COVID-19 Electrochemical Sensor}

Figure 1 showed a schematic diagram of the development of the electrochemical COVID-19 biosensor. First, Aucoated PDMS substrates $(10 \mathrm{~mm}$ width $\times 5 \mathrm{~mm}$ width $)$ were cleaned by rinsed with ethanol and dried under an $\mathrm{N} 2$ stream. Next, a $50 \mu \mathrm{l}$ of the antiCOVID-19 antibody $(10 \mu \mathrm{g} / \mathrm{ml})$ in a $10 \mathrm{mmol} \mathrm{L}^{-1}$ PBS buffer was added and kept at $4{ }^{\circ} \mathrm{C}$ overnight. Then, we rinsed the substrates three times with $10 \mathrm{mmol} \mathrm{L}^{-1} \mathrm{PBS}$ and dried them under nitrogen gas. Finally, $50 \mu \mathrm{l}$ of different concentrations of COVID-19 protein concentrations in $10 \mathrm{mmol} \mathrm{L}^{-1} \mathrm{PBS}$ were added to the substrates and incubated at $4{ }^{\circ} \mathrm{C}$ for a further $1 \mathrm{~h}$. After that, the substrate was washed three times with $10 \mathrm{mmol} \mathrm{L}^{-1} \mathrm{PBS}$ and DIW and dried under $\mathrm{N}_{2}$ gas. PCR was achieved using the SimpliAmp thermal cycler (Applied Biosystems, USA). The surface morphology of the Au substrate was analyzed by a scanning electron microscope (SEM) (ISI DS-130C, Akashi Co., Tokyo, Japan) and the atomic force microscopy (AFM).

\subsection{Topological Analysis by AFM}

The Au substrate surface's topography was investigated with an AFM inverted optical microscope (NTEGRA spectra, NT-MDT, Russia) with a semi-contact mode at room temperature under air conditioning. The maximum scan range of the system was $100 \mathrm{~mm}$ in both the $x$ and $y$ axes. The cantilevers were NSG01 type, which had a typical resonant frequency in the range of $115-190 \mathrm{kHz}$ and a force constant of $2.5-10 \mathrm{~N} / \mathrm{m}$, and the images were acquired at a scan rate of $1 \mathrm{~Hz}$ [38]. 


\subsection{Electrochemical Detecting of COVID-19 Concentrations}

The immobilization of the anti-COVID-19 antibody and its interaction with the COVID-19 protein was confirmed using the SERES signals. The SERS spectra were collected on Raman spectroscopy with a SENTERRA inverted confocal Raman microscope (Bruker Optics Inc., Germany). A CCD camera detection system and OPUS software were employed for data acquisition. Raman spectra were recorded using NIR laser emitting light at a wavelength of $785 \mathrm{~nm}$ and a power of $50 \mathrm{~mW}$ at the sample. Ten scans of $5 \mathrm{~s}$ from $200 \mathrm{~cm}^{-1}$ to $2000 \mathrm{~cm}^{-1}$ were recorded, and the mean of these scans was used.

The electrochemical measurements were achieved using the AUTOLAB electrochemical workstation instrument (Metrohm, Herisau, Switzerland). We have developed a three-electrode-based homemade electrochemical cell consisting of $\mathrm{Ag} / \mathrm{AgCl}$ as the reference electrode, Au microcuboid electrode as the working electrode, and Pt wire auxiliary electrode. In addition, PBS $(1 \times, \mathrm{pH}=7.4)$ was used as the electrolyte within the potential window from $-0.1 \mathrm{~V}$ to $+0.7 \mathrm{~V}$ at a scan rate of $50 \mathrm{mV} / \mathrm{s}$.

\subsection{Electrochemical Sensing of COVID-19 in a Real Sample Based on CV and SWV Techniques}

Here, we have used a real human sample that contains a COVID-19, which has been obtained from the nasal swab for direct detecting the COVID-19. About $50 \mu \mathrm{L}$ of the sample was directly immobilized antiCOVID-19 antibody/ Au micropattern substrate for about $6 \mathrm{~h}$ at room temperature. Then the substrate was directly used to investigate the COVID-19 based on CV and SWV techniques.

Acknowledgements This research is funded by King Abdulaziz City for Science and Technology (KACST), Riyadh, Saudi Arabia (Grant number: 5-20-01-031-0009). We gratefully acknowledge the financial support of the KACST.

\section{Declarations}

Conflict of interest The authors declare no competing financial interests.

\section{References}

1. Liu, D.X., Liang, J.Q., Fung, T.S.: Human Coronavirus-229E, -OC43, -NL63, and -HKU1 (Coronaviridae). Encycl. Virol. 2, 428-440 (2021)

2. Zaki, A.M., van Boheemen, S., Bestebroer, T.M., Osterhaus, A.D.M.E., Fouchier, R.A.M.: Isolation of a novel coronavirus from a man with pneumonia in Saudi Arabia. N. Engl. J. Med. 367, 1814-1820 (2012)

3. World Health Organization (WHO). Middle East respiratory syndrome coronavirus (MERS-CoV), MERS situation Update November 2019. WHO; 2020. Available from: www.who.int/ emergencies/mers-cov/en/ (2020).

4. Ministry of Health $(\mathrm{MOH})$. Command and Control Center. National Public Health Events, 2020. Ministry of Health; 2020.

5. World Health Organization (WHO). MERS situation update, January 2020. Available from:http://www.emro.who.int/pande mic-epidemic-diseases/mers-cov/mers-situation-updatejanuary2020.html.

6. https://www.worldometers.info/coronavirus/

7. Huang, C., et al.: Clinical features of patients infected with 2019 novel coronavirus in Wuhan, China. Lancet 395, 497-506 (2020)

8. Cui, F., Zhou, H.S.: Diagnostic methods and potential portable biosensors for coronavirus disease 2019. Biosens. Bioelectron. 165, 112349 (2020)

9. Wu, F., et al.: A new coronavirus associated with human respiratory disease in China. Nature 579, 265-269 (2020)

10. Wang, D., et al.: A multiplex ELISA-based protein array for screening diagnostic proteins and diagnosis of Flaviviridae infection. Eur. J. Clin. Microbiol. Infect. Dis. 34, 1327-1336 (2015)

11. El-Said, W.A., Choi, J.-W.: Electrochemical biosensor consisted of conducting polymer layer on gold nanodots patterned Indium Tin Oxide electrode for rapid and simultaneous determination of purine bases. Electrochim. Acta. 123, 51-57 (2014)

12. Asad, M., et al.: Orange peel derived $\mathrm{C}$-dots decorated $\mathrm{CuO}$ Nanorods for the selective monitoring of Dopamine from deboned chicken. Electroanalysis 32, 11-18 (2020)

13. Khalifa, M.M., et al.: Highly specific Electrochemical Sensing of Pseudomonas aeruginosa in patients suffering from corneal ulcers: a comparative study. Sci. Rep. 9, 18320 (2019)

14. Kim, T.-H., El-Said, W.A., An, J.H., Choi, J.-W.: ITO/gold nanoparticle/RGD peptide composites to enhance electrochemical signals and proliferation of human neural stem cells. Nanomedicine 9, 336-344 (2013)

15. El-Said, W.A., Yea, C.-H., Choi, J.-W., Kwon, I.-K.: Ultrathin polyaniline film coated on an indium-tin oxide cell-based chip for study of anticancer effect. Thin. Solid. Films. 518, 661-667 (2009)

16. Shin, J.-W., et al.: Silver nanoparticle modified electrode covered by graphene oxide for the enhanced electrochemical detection of dopamine. Sensors 17, 2771 (2017)

17. Li, R., et al.: A flexible and physically transient electrochemical sensor for real-time wireless nitric oxide monitoring. Nat. Commun. 11, 3207 (2020)

18. El-Said, W.A., Kim, T.-H., Chung, Y.-H., Choi, J.-W.: Fabrication of new single cell chip to monitor intracellular and extracellular redox state based on spectroelectrochemical method. Biomaterials 40, 80-87 (2015)

19. Senel, M., Dervisevic, M., Alhassen, S., Alachkar, A., Voelcker, N.H.: Electrochemical micropyramid array-based sensor for in situ monitoring of dopamine released from neuroblastoma cells. Anal. Chem. 92, 7746-7753 (2020)

20. El-Said, W.A., Lee, J.-H., Oh, B.-K., Choi, J.-W.: Electrochemical sensor to detect neurotransmitter using gold nano-island coated ITO electrode. J. Nanosci. Nanotechnol. 11, 6539-6543 (2011)

21. El-Said, W.A., Yea, C.-H., Kwon, I.-K., Choi, J.-W.: Fabrication of electrical cell chip for the detection of anticancer drugs and environmental toxicants effect. BioChip. J. 3, 105-112 (2009)

22. Abbas, Y., et al.: Heteroatom-doped Carbon nanoparticle-ionic liquid composites as electrochemical sensors for Uric acid. ACS. Appl. Nano. Mater 3, 11383-11390 (2020) 
23. Yilmaz, D., et al.: Application of Raman spectroscopy in monoclonal antibody producing continuous systems for downstream process intensification. Biotechnol. Prog. 36, e2947 (2020)

24. Wen, Z.-Q.: Raman spectroscopy of protein pharmaceuticals. J. Pharm. Sci. 96, 2861-2878 (2007)

25. Ashton, L., Blanch, E.W.: pH-induced conformational transitions in a-lactalbumin investigated with two-dimensional Raman correlation variance plots and moving windows. J. Mol. Struct. 974, 132-138 (2010)

26. McAvan, B.S., et al.: Raman spectroscopy to monitor post-translational modifications and degradation in Monoclonal antibody therapeutics. Anal. Chem. 92, 10381-10389 (2020)

27. Baker, A.E., Mantz, A.R., Chiu, M.L.: Raman spectroscopy characterization of antibody phases in serum. mAbs 6, 1509-1517 (2014)

28. Ettah, I., Ashton, L.: Engaging with Raman spectroscopy to investigate antibody aggregation. Antibodies 7, 24 (2018)

29. Baker, A.N., et al.: The SARS-COV-2 spike protein binds sialic acids and enables rapid detection in a lateral flow point of care diagnostic device. ACS. Cent. Sci. 6, 2046-2052 (2020)

30. Moitra, P., Alafeef, M., Dighe, K., Frieman, M., Pan, D.: Selective naked-eye detection of SARS-CoV-2 mediated by N gene targeted antisense oligonucleotide capped plasmonic nanoparticles. ACS. Nano. 14, 7617-7627 (2020)

31. Jiao, J., et al.: DNA nanoscaffold-based SARS-CoV-2 detection for COVID-19 diagnosis. Biosens Bioelectron 167, 112479 (2020)

32. Ji, T., et al.: Detection of COVID-19: a review of the current literature and future perspectives. Biosens Bioelectron 166, 112455 (2020)
33. Mertens, P., et al.: Development and potential usefulness of the COVID-19 Ag Respi-Strip diagnostic assay in a pandemic context. Front. Med. 7, 225 (2020)

34. Ali, Md.A., et al.: Sensing of COVID-19 antibodies in seconds via Aerosol Jet Nanoprinted reduced-Graphene-oxide-coated 3D electrodes. Adv. Mater. 33, 2006647 (2021)

35. Shi, L., et al.: Development of SPR biosensor for simultaneous detection of multiplex respiratory viruses. Biomed. Mater. Eng. 26, S2207-S2216 (2015)

36. Lee, T., et al.: Recent advances in AIV biosensors composed of Nanobio hybrid material. Micromachines 9, 651 (2018)

37. Layqah, L.A., Eissa, S.: An electrochemical immunosensor for the corona virus associated with the Middle East respiratory syndrome using an array of gold nanoparticle-modified carbon electrodes. Microchim. Acta. 186, 224 (2019)

38. El-Said, W.A., Yea, C.-H., Jung, M., Kim, H., Choi, J.-W.: Analysis of effect of nanoporous alumina substrate coated with polypyrrole nanowire on cell morphology based on AFM topography. Ultramicroscopy 110(6), 676 (2010)

Publisher's Note Springer Nature remains neutral with regard to jurisdictional claims in published maps and institutional affiliations. 